\title{
Effective drug release from safe ultrasound-triggered nanocarriers
}

Matthew G. Wilson ${ }^{1}$ and Jan Kubanek ${ }^{1}$

${ }^{1}$ Department of Biomedical Engineering, University of Utah, 36 S Wasatch Dr, Salt Lake City, UT 84112

Selective delivery of medication into specified tissue targets would realize the promise of personalized medicine with minimal side effects. Such an approach could be particularly transformative for patients with brain disorders, in whom drugs could be released in the impaired brain circuits at high concentration while sparing other brain regions and organs. Focused ultrasound provides noninvasive and practical means to release drugs from nanocarriers selectively at its target. However, which nanoparticle formulations provide safe and effective release and under which ultrasound parameters has been unclear. To expedite regulatory approval, we tested release effectiveness from nanocarriers filled with perfluorocarbon cores of relatively high boiling points (up to $142^{\circ} \mathrm{C}$ ). We confirmed the safety of these nanocarriers in non-human primates. Crucially, we found that these safe, high-boiling-point nanocarriers can be used for effective release so long as they are activated by ultrasound of frequencies lower than those used previously $(300 \mathrm{kHz})$. This study informs the formulation and release parameters for safe and effective drug delivery in specific parts of the body or brain regions.

Keywords: focused ultrasound, parameters, nanoparticles, boiling point, effective and safe release

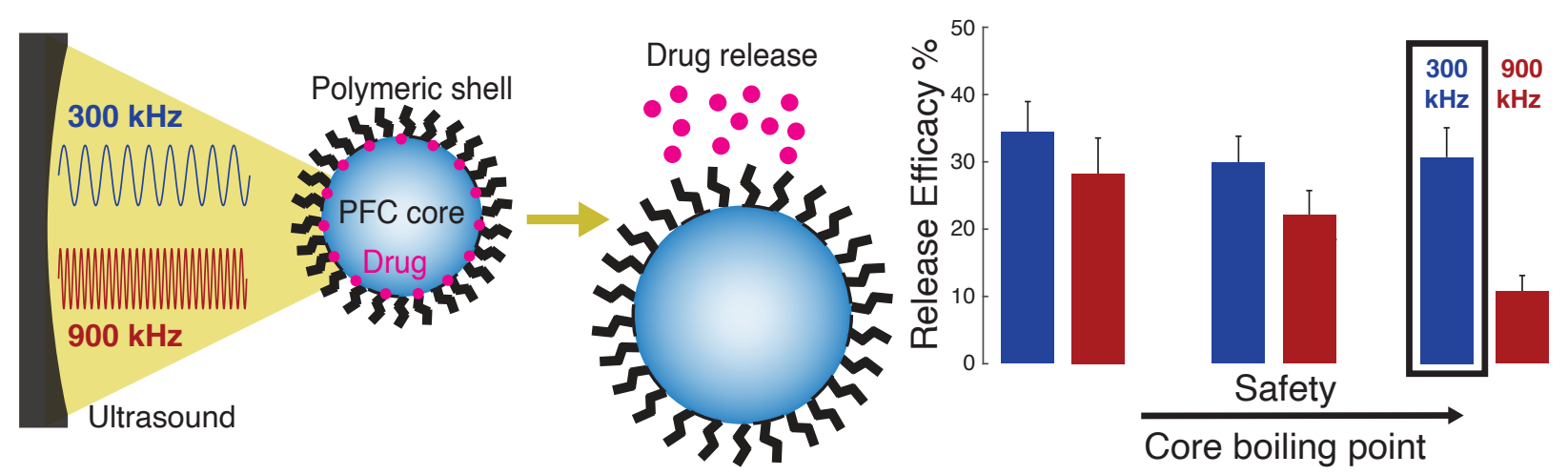

Drugs can be effectively released from ultrasound-sensitive nanoparticles filled with safe, high-boiling point cores so long as they are activated by low-frequency ultrasound.

\section{Introduction}

Treatments of mental and neurological disorders are often curbed by low effectiveness of drugs or their intolerable side effects (Bystritsky, 2006; Lyons and Pahwa, 2004; Louis et al., 2010; Zesiewicz et al., 2010; Al-Harbi, 2012; Elias et al., 2013; Elias et al., 2016; Jaffe et al., 2019). Consequently, millions of patients with disorders of brain function remain resistant to treatments and suffer from poor quality of life. There 


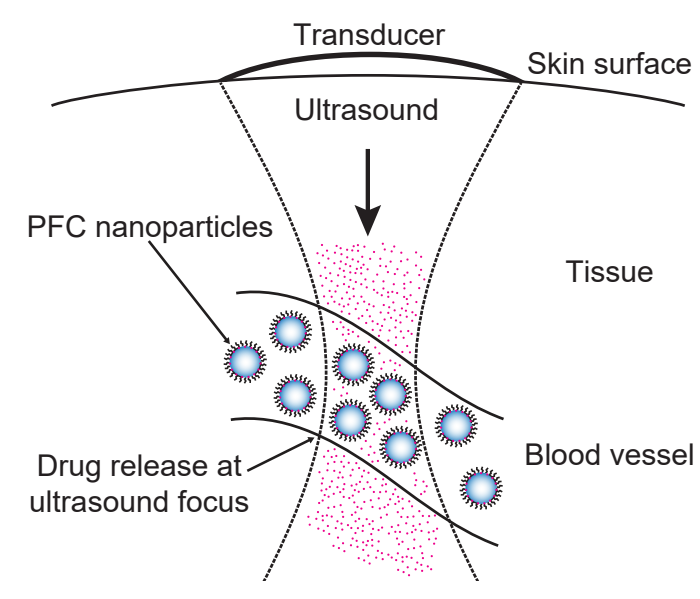

Figure 1. Controlled drug release in a specific body location. Drug-filled biodegradable nanoparticles are injected into the bloodstream. Brief pulses of focused, low-intensity ultrasound are applied from the outside of the body using a standard transducer. At the ultrasound target, the focused pressure wave mechanically stimulates the nanoparticles, which leads to a release of the drug specifically at the target and not elsewhere.

is a critical need for approaches that deliver medication selectively in the desired neural circuits and at a high concentration.

Pioneering work in this domain devised temperature-sensitive liposomes (e.g., Needham and Dewhirst, 2001) that can be activated by heat or radiation. However, localized heating at depth has been challenging to achieve safely and in a controlled manner. Recent efforts have shifted to using ultrasound as a safe and practical form of energy for localized drug release. Ultrasound can be controllably focused at depth into specific tissue targets using inexpensive hardware. Targeting systemically injected drug carriers, ultrasound can trigger focal release with minimal off-target effects (Figure 1).

Several groups have shown that ultrasound can release drugs from nano-sized structures stabilized with biocompatible shells (Rapoport et al., 2007; Rapoport et al., 2009; Rapoport et al., 2011; Rapoport et al., 2015; Rapoport, 2016; Airan et al., 2017; Boissenot et al., 2017; Airan et al., 2017; Wang et al., 2018; Zhong et al., 2019; Wu et al., 2018; Lea-Banks and Hynynen, 2021; Lea-Banks et al., 2021; Somaglino et al., 2021). These nano-sized structures have been commonly filled with perfluorocarbon (PFC) cores. PFCs are highly inert and bestow the resulting nanoparticles with sensitivity to ultrasound. When exposed to ultrasound, the PFC core has been hypothesized to change phase from liquid to gas, greatly expanding in volume, and thus mediating drug release (Sheeran et al., 2013; Doinikov et al., 2014; Shpak et al., 2014; Rapoport, 2016; Kripfgans et al., 2000). Harnessing this understanding, a majority of previous studies used nanoparticles with PFC boiling points below body temperature (Rapoport et al., 2007; Rapoport et al., 2009; Rapoport et al., 2011; Sheeran et al., 2012; Rapoport et al., 2015; Rapoport, 2016; Airan et al., 2017; Airan et al., 2017; Wang et al., 2018; Zhong et al., 2019; Wu et al., 2018; Lea-Banks and Hynynen, 2021; Lea-Banks et al., 2021). From safety perspective, however, the use of PFCs with higher boiling point is preferable to prevent a risk of embolism. For instance, FDA approvals have been readily granted to PFCs with high boiling points to be administered into humans in large quantities (Castro and Briceno, 2010; $\mathrm{Li}$ et al., 2018). Nonetheless, drug-release from such nanoparticles appears to be less effective (Boissenot et al., 2017; Somaglino et al., 2021). In this study, we tested whether such high-boiling-point-based nanoparticles could be activated with ultrasound of particular parameters. If so, the specific conjuction of nanoparticle formulation and ultrasound parameters could provide treatments that are both effective and safe. 


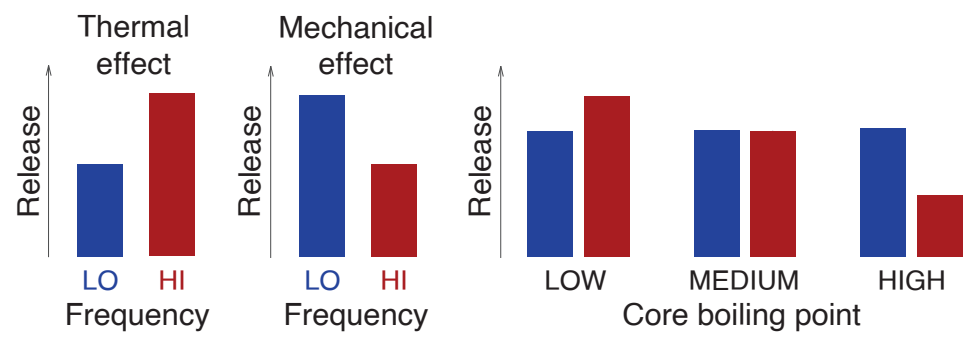

Figure 2. Study design and predictions. The release should increase/decrease with the ultrasound frequency under a ther$\mathrm{mal} /$ mechanical effect (left). A thermal effect, such as the predominantly hypothesized phase change, should be accentuated by PFCs with lower boiling points (right, red).

We based this study on two hypotheses. First, drug release from PFC-based nanoparticles may involve mechanical or thermal effects (Rapoport et al., 2010; Rapoport, 2016; Cao et al., 2018; Lea-Banks et al., 2019). The ultrasound frequency can engage these two mechanisms differentially, with high frequencies more likely to engage thermal mechanisms by depositing more energy at the target. Low frequencies are more likely to induce mechanical effects including particle displacement, cavitation, or vaporization of dissolved gases in the PFC core (Figure 2, left). Second, according to the predominant hypothesis of release, ultrasound delivered at the target induces vaporization of the PFC core (Sheeran et al., 2013; Doinikov et al., 2014; Shpak et al., 2014; Rapoport, 2016). This implies that the PFC boiling point should be inversely related to release effectiveness and that this effect will be mainly observed at high ultrasound frequencies (Figure 2, right).

\section{Results}

\section{In vitro ultrasound-triggered drug release}

We prepared PFC-based, copolymer-stabilized nanoparticles using a similar approach as described in previous studies (see Materials and Methods for details). We loaded the nanoparticles with the neuromodulatory drug propofol (Orser et al., 1994; Wang et al., 2018) and quantified the effectiveness of its release using an approach described previously (Zhong et al., 2019). Uniquely, we tested how the critical component of the nanoparticle-its core-governs the amount of drug released as a function of specific ultrasound parameters. Specifically, we tested the release effectiveness of three different PFC cores-perfluoropentane (PFP), decafluoropentane (DFP), and perfluorooctylbromide (PFOB). These PFCs have boiling points of $29^{\circ} \mathrm{C}, 55^{\circ} \mathrm{C}$, and $142^{\circ} \mathrm{C}$, respectively. Nanoparticles with these cores had comparable sizes: mean \pm SD of $543.3 \pm 23.7,550.8 \pm 91.7$, and $473.0 \pm 28.4 \mathrm{~nm}$ for PFP, DFP, and PFOB-based nanoparticles, respectively.

We used an ultrasonic transducer capable of operating at both low $(300 \mathrm{kHz})$ and high $(900 \mathrm{kHz})$ frequencies (Riis and Kubanek, 2021). We focused the ultrasonic beam on vials containing particular nanoparticle samples and tested the effects of specific ultrasound parameters on drug release. We found (Figure 3, left) that ultrasound of low frequency $(300 \mathrm{kHz})$ triggered more drug release from the nanoparticles than ultrasound of high frequency $(900 \mathrm{kHz})$. The difference in the percentage of

drug released at the two frequencies (31.7\% and $20.3 \%$, respectively) was significant $(t(130)=3.3$, $p=0.0013$, two-sample two-tailed t-test). In line with our hypothesis, we found (Figure 3, right) that at the higher $900 \mathrm{kHz}$ frequency, the release effectiveness strongly depends on the boiling point of the PFC 

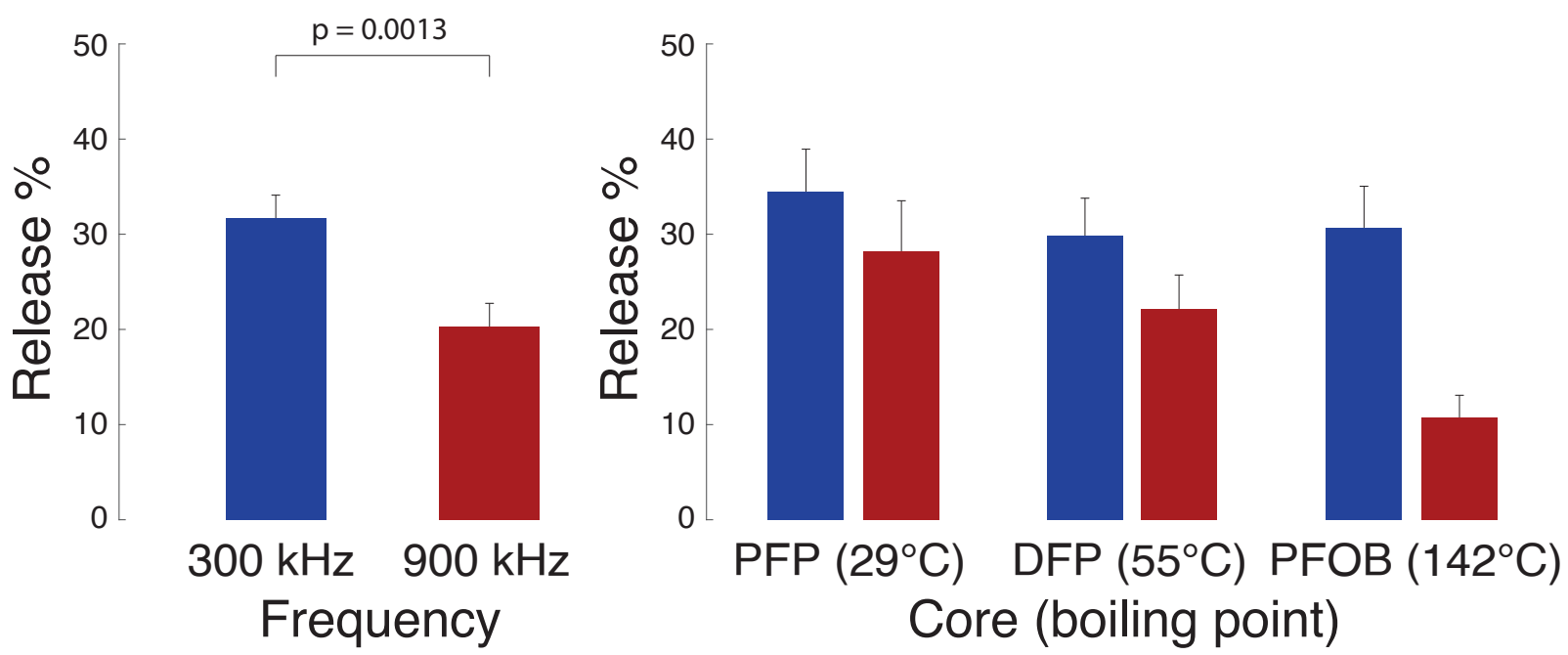

Figure 3. Release from nanoparticles with distinct cores under two ultrasound frequency modes. Mean \pm s.e.m. propofol release relative to the amount encapsulated (see Materials and Methods for details) for the two ultrasound frequencies (left) and the three cores (right) tested. The ultrasound was delivered in $100 \mathrm{~ms}$ pulses repeated 60 times over the period of 1 minute. The $p$-value denotes the significance of a two-sample two-tailed t-test. A complete statistical analysis of the effects is provided in Table 1.

core. The scaling is in the expected direction - the lower the boiling point of the PFC, the higher the release effectiveness. An omnibus ANOVA model (Table 1) that incorporated all factors tested (core, ultrasound frequency, ultrasound pressure) as well as all possible interactions, detected a significant core $\times$ frequency interaction $(F(2,90)=8.05, p=0.00061)$.

The scaling of the release effectiveness by the PFC boiling point (red bars in Figure 3, right) suggests the engagement of a thermal mechanism at the higher frequency, in agreement with previous propositions (Wu et al., 2018; Doinikov et al., 2014; Rapoport, 2016; Zhang et al., 2010). If this is the case, the release should also scale with the average ultrasound intensity $I$ delivered into the nanoparticles. For longitudinal waves, it holds $I=\frac{P^{2}}{2 Z}$, where $P$ is the ultrasound pressure amplitude, and $Z$ is the acoustic impedance of the medium (Cobbold, 2006). Thus, a thermal effect scales with pressure squared. We indeed found that the data at $900 \mathrm{kHz}$ showed a quadratic dependence on pressure (Figure 4, right). Specifically, quadratic fits to the data (solid curves) explained 96.5, 93.5, and $94.8 \%$ of the variance in the PFP, DFP, and PFOB data points. In comparison, linear fits only explained $80.3,80.5$, and $69.3 \%$ of the variance, respectively. The difference in the mean variance explained by the quadratic and linear fits ( $94.9 \%$ versus $76.7 \%)$ was significant $(t(2)=4.83, p=0.040$, two-sided t-test). In contrast, the lower $300 \mathrm{kHz}$ frequency showed a linear dependence of the release on pressure (Figure 4, left). A quadratic fit did not significantly $(p>0.16)$ differ from the linear fit in terms of variance explained $(89.5 \%$ versus $89.3 \%$ ). The linear dependence on pressure at $300 \mathrm{kHz}$ is consistent with a mechanical rather than a thermal effect (see Discussion for details).

We summarize the effects of the three factors tested (core, ultrasound frequency, and ultrasound pressure) as well as all possible interactions in an omnibus ANOVA model (Table 1). This analysis confirms that both the core and ultrasound parameters (frequency and pressure) are important considerations for 

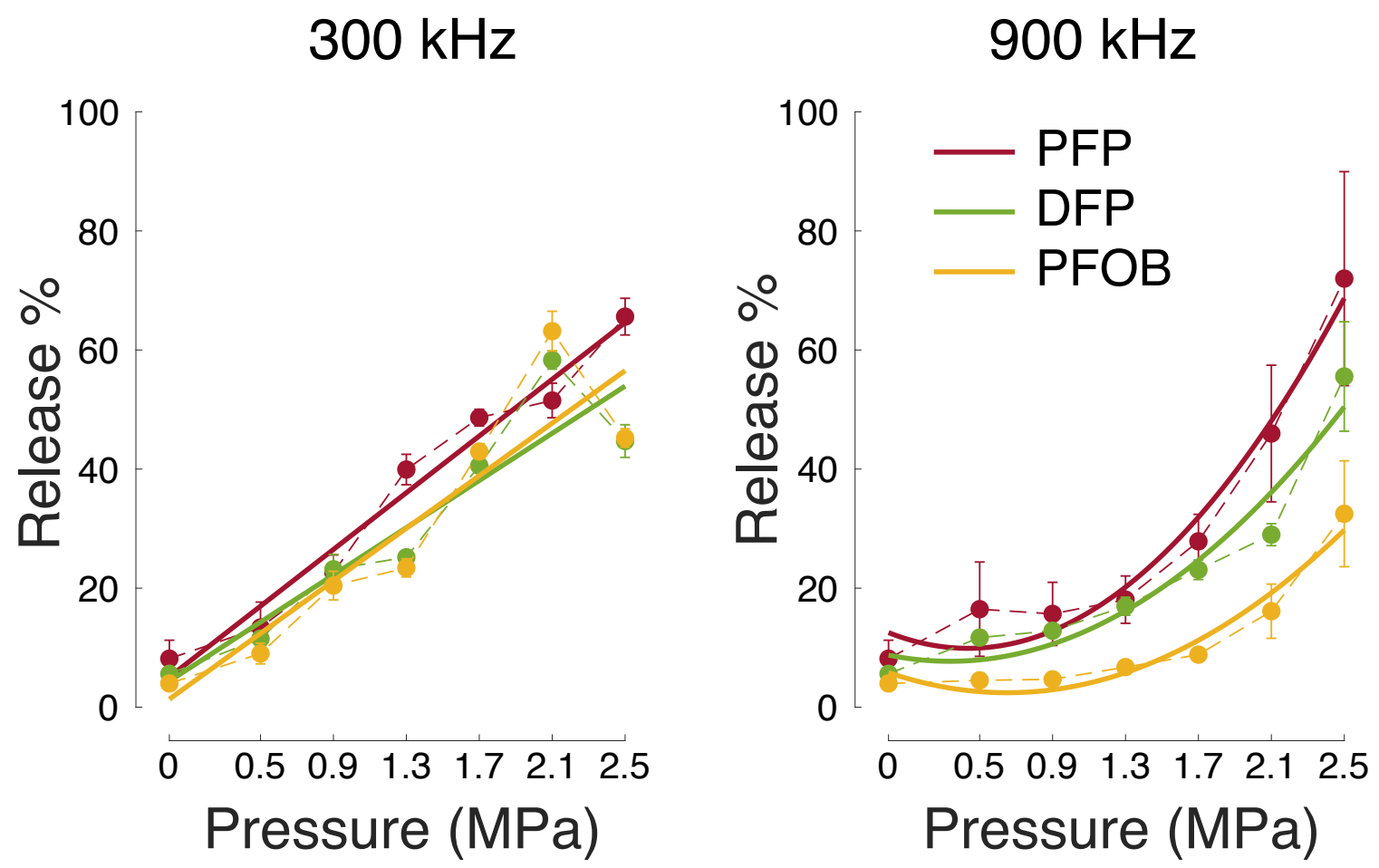

Figure 4. Release across all tested factors. Mean \pm s.e.m. percentage of the released propofol for the two ultrasound frequencies (left versus right), ultrasound pressure amplitude (abscissa), and the three cores tested (color; see inset). The thick lines represent quadratic fits to the data. Notably, for the $300 \mathrm{kHz}$ data, linear fits were as explanatory as the quadratic fits (see text). 


\begin{tabular}{l|l} 
Factor & Significance \\
\hline \hline Core & $<\mathbf{0 . 0 0 1}$ \\
$F$ & $<\mathbf{0 . 0 0 1}$ \\
$P$ & $<\mathbf{0 . 0 0 1}$ \\
\hline Core $\times F$ & $<\mathbf{0 . 0 0 1}$ \\
$F \times P$ & $<\mathbf{0 . 0 0 1}$ \\
Core $\times P$ & $\mathbf{0 . 0 3 0}$ \\
Core $\times F \times P$ & $\mathbf{0 . 0 4 9}$ \\
\hline
\end{tabular}

Table 1. Summary of the effects. The effects of the nanoparticle core, ultrasound frequency $(F)$, and ultrasound pressure $(P)$. These effects were assessed using a three-way ANOVA that featured the three main effects and all possible interactions. Bold entries are significant $(p<0.05)$.

effective release. In addition, the factors interact: the selection of a specific core must be performed in conjunction with suitable ultrasound parameters.

From safety perspective, cores with higher boiling points should be more stable, thus minimizing the risk of embolism and spontaneous drug release when injected into the bloodstream. Indeed, we observed a spontaneous rate of release (0 MPa data point in Figure 4) of just $4.0 \%$ for PFOB nanoparticles compared to $8.2 \%$ for PFP. As noted above, the more stable, PFOB-based nanoparticles can be as effective as PFP-based nanoparticles when driven at the lower, $300 \mathrm{kHz}$ frequency. Henceforth, we assessed the safety, blood clearance kinetics, and organ biodistribution specifically for the PFOB-based nanoparticles. These tests involved a macaque monkey, a marmoset monkey, and 3 Sprague-Dawley rats.

\section{In vivo safety studies}

The injection of the propofol-filled PFOB nanoparticles into the bloodstream of the animals did not cause notable changes in vital signs. In particular, Figure 5 shows the heart rate as a function of time, aligned to the time of the bolus administration. There was no significant difference between the heart rate measured during a 5-minute interval immediately prior to the bolus and the last 5 minutes of the measurements (217.7 compared with 218.9 beats per minute, $t(5)=-0.16, p=0.88$, paired two-sided $\mathrm{t}$-test). The vital signs of the macaque monkey were particularly steady.

We next investigated the pharmacokinetics of the nanoparticles in the macaque monkey. To do so, we incorporated a fluorescent dye in the nanoparticles along with propofol (see Materials and Methods). While the macaque was anesthetized, we drew blood samples at 2, 10, 20, 40, 80, and 120 minutes following injection. We then quantified the amount of fluorescence from these blood samples relative to the 2 min time point (Figure 6). This blood clearance curve has a relative half-life of 32 minutes, similar to previous reports with PFP-based nanoparticles in rats (Airan et al., 2017; Cao et al., 2018). An accurate fit to these data could only be made by superimposing two exponentials, one with a fast and one with a slow rate of decay (Figure 6). This suggests that the clearance of the nanoparticles involved two distinct processes or organs.

Finally, we assessed in which organs the PFOB-based nanoparticles degrade. Immediately following the measurements of vital signs (Figure 5), the animals were sacrificed and their major organs extracted for analysis. The nanoparticles were found predominantly in the liver, in both the primates and rats (Figure 7 ). The kidneys were the only other organs where average relative fluorescence exceeded $7 \%$. These findings resemble those obtained using PFP in rats (Wang et al., 2018; Cao et al., 2018). 


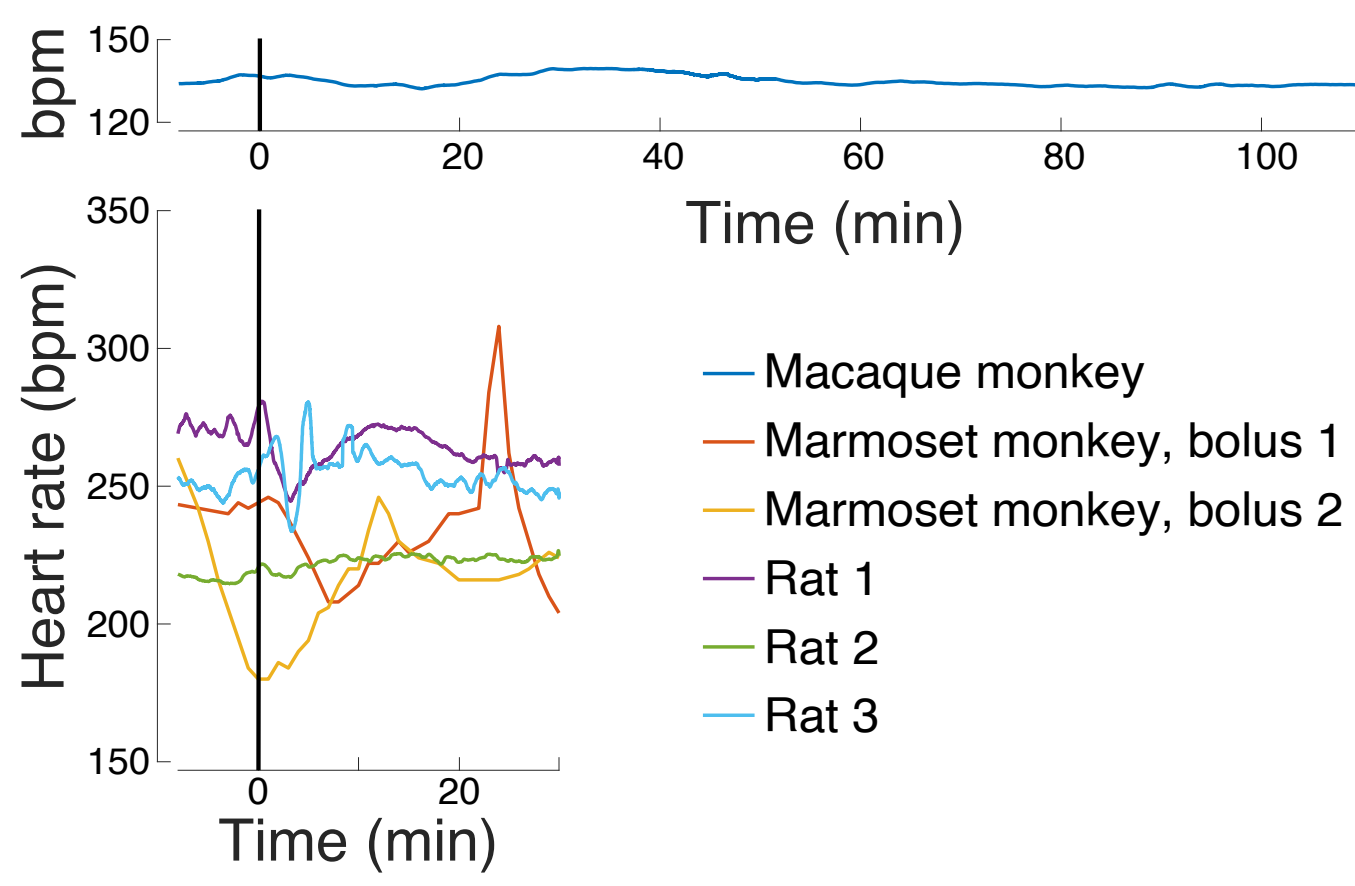

Figure 5. Safety of PFOB-based nanoparticles. The heart rate of a macaque monkey, marmoset monkey, and 3 rats before and after intravenous injections of PFOB-based nanoparticles. The nanoparticles were filled with propofol at a concentration of $1 \mathrm{mg} / \mathrm{kg}$ (macaque), $1 \mathrm{mg} / \mathrm{kg}$ (marmoset), and 0.5, 1 , and $1 \mathrm{mg} / \mathrm{kg}$ (rats). The injected volumes were $5 \mathrm{ml}$ (macaque), $2 \mathrm{ml}$ (marmoset), and 0.48,0.53, and $1.1 \mathrm{ml}$, respectively (rats). In the marmoset, we performed two injections separated by 45 minutes. The EKG was recorded using a portable EKG-monitoring system. The heart rate in the smaller animals (marmoset and rats) tracked the frequently varying levels of isoflurane anesthesia-higher levels led to lower heart rate and reversely.

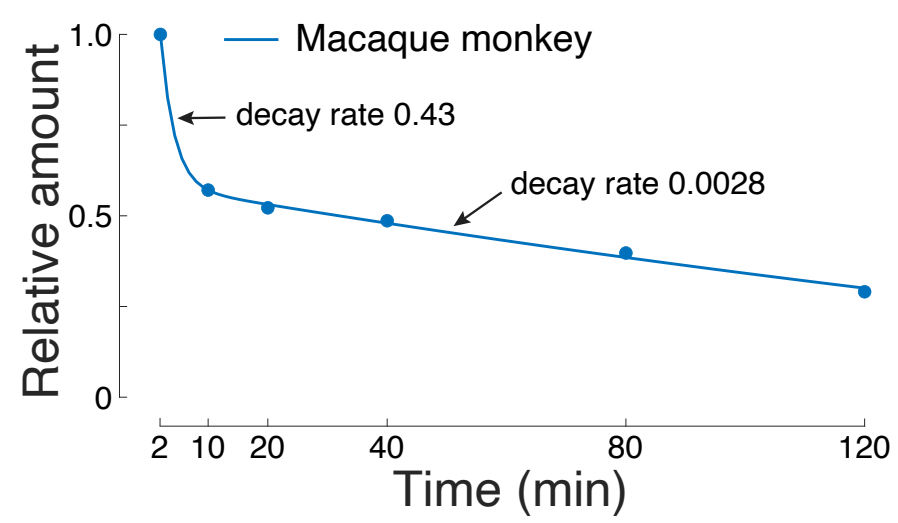

Figure 6. Blood clearance kinetics in macaque monkey. Relative fluorescence as a function of specific sampling times indicated on the abscissa. The PFOB-based nanoparticles contained propofol and an infrared dye (IR800RS, LI-COR). The nanoparticles were injected via a saphenous vein catheter at time 0 at a volume of $5 \mathrm{ml}$. Blood samples $(1 \mathrm{ml}$ each) were taken at the times indicated on the abscissa and subjected to fluorescence analysis (see Materials and Methods). The data points were captured using the superposition of two exponentials. One showed a fast and the other a slow time constant (see inset). 


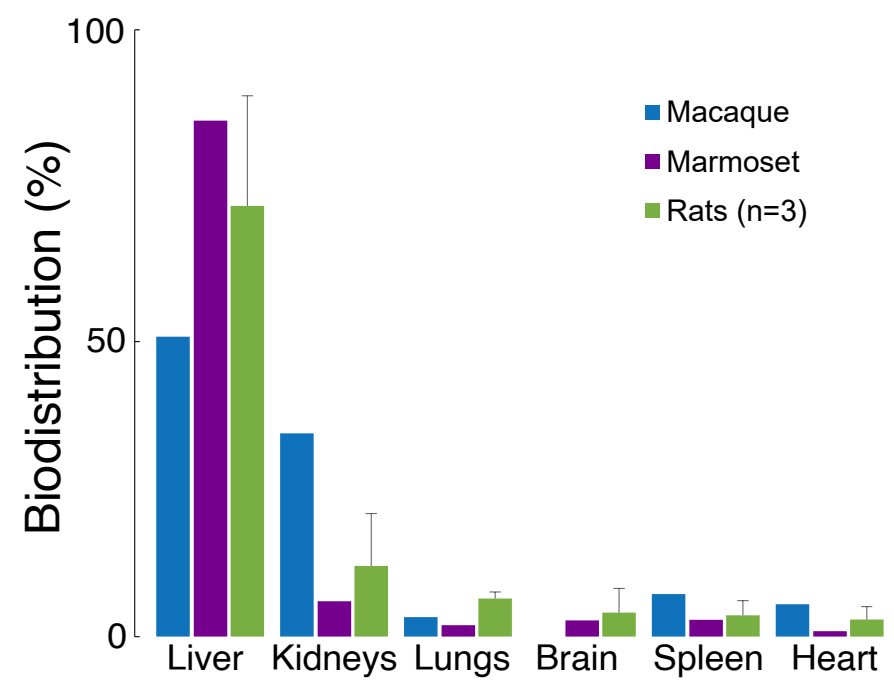

Figure 7. Biodegradation in major organs. Distribution of PFOB-based nanoparticles in major organs of the marmoset and the rats. The presence was assessed using the same infrared dye as in Figure 6. The figure shows the percentage of total fluorescence (and hence, the relative accumulation of the nanoparticles) measured within the respective organs. The rat data are presented as means \pm standard deviation.

\section{Discussion}

This study investigated the effectiveness and safety of drug release from biocompatible nanoparticles triggered by focused ultrasound. Uniquely, we evaluated the release efficacy from nanoparticles filled with PFCs with distinct boiling points, including boiling points above the body temperature, and investigated the relevant ultrasound parameters that activate them. We found that low ultrasound frequencies (300 kHz in particular) are more effective in mediating release in general and that low frequencies are paramount for effective release from PFCs with a high boiling point. Moreover, we validated the nanoparticles' safety and blood clearance kinetics with a high boiling point in non-human primates and noted no safety issues.

The core has been hypothesized to be a critical factor in governing the effectiveness of ultrasoundbased drug release (Wu et al., 2018; Rapoport et al., 2011; Somaglino et al., 2021). Indeed, our $900 \mathrm{kHz}$ data show (Figure 3, right, red) that at the range of commonly applied ultrasound frequencies $(\geq 1 \mathrm{MHz})$, the core is a key determinant of release effectiveness. We found that the higher the boiling point of the encapsulated PFC, the lower the release effectiveness. This finding is in accord with previous results (Wu et al., 2018; Doinikov et al., 2014; Rapoport, 2016; Zhang et al., 2010). Critically, we found that lowering the frequency to $300 \mathrm{kHz}$ increased the release effectiveness at a general, core-independent level (Figure 3). This effect can also be found in a study that used PFP (Zhong et al., 2019). The application of low-frequency ultrasound thus opens the path to using high-boiling-point cores (e.g., PFOB) as release actuators. A frequency of $300 \mathrm{kHz}$ provides spatial resolution on the order of several millimeters, which approximates the focal volume of the drug released in tissue by ultrasound (Wang et al., 2018; Lea-Banks and Hynynen, 2021).

The majority of studies that applied ultrasound to PFC-based nanoparticles used PFCs with boiling point below the body temperature (Rapoport et al., 2007; Rapoport et al., 2009; Rapoport et al., 2011; Sheeran et al., 2012; Rapoport et al., 2015; Rapoport, 2016; Airan et al., 2017; Airan et al., 2017; Wang et al., 2018; Zhong et al., 2019; Wu et al., 2018; Lea-Banks and Hynynen, 2021; Lea-Banks et al., 2021). Although the Laplace pressure of sub-micron particles can effectively increase the boiling point 
(Rapoport, 2012), PFCs with low boiling points may suffer from instability issues, which may raise safety concerns. For example, our data show that PFP-based nanoparticles not exposed to ultrasound (0 MPa datapoint in Figure 4) have twice the rate of spontaneous release compared with PFOB. Further, low boiling point PFCs can form persistent microbubbles after vaporization by ultrasound (Rapoport, 2016; Rapoport et al., 2009; Lea-Banks et al., 2021). In fact, with respect to PFC safety, the FDA has thus far approved PFCs with a high boiling point for large-scale use in humans-PFOB in particular. Specifically, PFOB has been used in liter- quantities in humans as agents that deliver oxygen into tissues. The PFOBbased products have included LiquiVent-an oxygen-carrying liquid drug, and Oxygent (both Alliance Pharmaceutical Corporation, San Diego, CA, USA)—a blood substitution agent (Cohn and Cushing, 2009; Castro and Briceno, 2010; Li et al., 2018). Our finding that PFOB can be used for effective release from nanoparticles at low ultrasound frequencies (Figure 3, Figure 4) should mitigate the boiling-point associated concerns. Indeed, we administered PFOB-based nanoparticles to two non-human primates and three rats (Figure 5) and detected no changes in vital signs.

We evaluated the pharmacokinetics of blood clearance of the PFOB-based nanoparticles in a macaque monkey. The nanoparticles showed a half-life of $32 \mathrm{~min}$ in the primate (Figure 6). This is on the order of the values reported in other studies that used PEGylated nanoparticles (Airan et al., 2017; Cao et al., 2018). The half-life can be controlled by the type and chain length of the copolymers constituting the shell (Rapoport et al., 2015). Various copolymer shells, and PEG-based copolymers in particular, have been approved by the FDA for a variety of indications (Bobo et al., 2016).

The monkey blood clearance data were captured with two decaying exponentials (Figure 6). The dual exponential decay was similar to that reported using PFP-based particles in rats (Airan et al., 2017), and suggests an involvement of two distinct clearance processes or organs. In a marmoset, macaque, and three rats, we found that PFOB-based nanoparticles were primarily degraded in the liver, with notable traces also observed in the kidneys (Figure 7). This finding aligns with those established using PFC-based nanoparticles in rats (Wang et al., 2018; Cao et al., 2018). Whether these two organs could produce the dual nature of the clearance kinetics observed in Figure 6 should be tested in future studies. Lungs may also contribute to the clearance (Airan et al., 2017; Cao et al., 2018). In the liter quantities introduced into humans, PFOB can be eliminated from the body in large part through exhalation (Krafft, 2001).

The in vitro release data presented here contribute to the understanding of the release mechanism. Thus far, the predominant hypothesis of action has been the vaporization of the PFC droplet upon the impact of focused ultrasound (Sheeran et al., 2013; Shpak et al., 2014; Doinikov et al., 2014; Wu et al., 2018; Rapoport et al., 2011; Somaglino et al., 2021). In this mechanism, the thermal and mechanical aspects of propagating ultrasound exceed the vaporization threshold governed by the PFC boiling point and the Laplace pressure. The PFC core increases in size (up to a 5-fold increase in diameter (Rapoport, 2012)), which contributes, in addition to any superimposed effects of ultrasound, to the drug release. Indeed, our data at the common range of ultrasound frequencies, $900 \mathrm{kHz}$, provide two lines of evidence supporting this hypothesis. First, we found that the release increases with decreasing boiling point (Figure 3, right), as expected from the vaporization hypothesis. Second, thermal energy delivered by ultrasound is known to be proportional to pressure squared (Cobbold, 2006). We indeed found a 
quadratic dependence of the release on pressure (Figure 4, right).

However, our (Figure 3, left) and a previous study (Zhong et al., 2019) suggest that ultrasound of frequencies lower than those in the common diagnostic range may mediate more effective drug release. Lower frequencies are known to accentuate mechanical effects, which can take two forms. First, ultrasound can induce cavitation, the formation of gaseous nuclei from dissolved gasses under the low-pressure epochs of the ultrasound pressure wave (Apfel and Holland, 1991). Cavitation can provide useful mechanical forces until it exceeds a threshold at which the formed gaseous nuclei collapse and cause damage (Apfel and Holland, 1991). From the FDA's 510(k) Track 3 standpoint (FDA, 2019), cavitation is unlikely to occur for mechanical index-defined as ultrasound pressure divided by the square root of frequency—values below 1.9. In our hands, a $300 \mathrm{kHz}, 1.0 \mathrm{MPa}$ pressure at target yields a mechanical index of 1.83. Despite being below the value of 1.9 , this pressure level already drives significant release (Figure 4, left). Thus, if cavitation is involved, it is either in the safe range or just one of several mechanisms. Second, PFCs are known to strongly bind oxygen (Cohn and Cushing, 2009; Castro and Briceno, 2010; Li et al., 2018). The negative pressure of the ultrasonic wave can produce an outward motion of the dissolved gas. The resulting forces are likely to mechanically distort the shell of the nanoparticle on a cycle-by-cycle basis and thus perpetuate release (Emanuel and Buchachenko, 1987; Rapoport et al., 2011). Whether mediated by oxygen or other molecules, the maximal displacement of a particle in the ultrasound path, $\xi_{m}$, is linearly proportional to the ultrasound pressure amplitude $P$ : $\xi_{m}=\frac{P}{Z} \int_{0}^{T / 2} \sin (2 \pi F t) d t=\frac{P}{Z \pi F}$, where $F$ is the ultrasound frequency and $Z$ the acoustic impedance. Indeed, our $300 \mathrm{kHz}$ data show a linear dependence of the release on pressure (Figure 4, left). This mechanism is supported by high-speed imaging, which did not detect a persistent phase change despite effective release in rodents (Zhong et al., 2019). Together, our findings indicate that both thermal and mechanical effects can be at play, depending on the applied ultrasound frequency. Higher frequencies accentuate thermal effects (PFC vaporization), whereas lower frequencies accentuate mechanical effects (cavitation or cycle-by-cycle particle displacement).

Interestingly, at $300 \mathrm{kHz}$, we observed a marginal decrease in drug release from DFP and PFOB nanoparticles at the highest ultrasound pressure tested (Figure 4, left). This effect has been observed in another study (Lea-Banks and Hynynen, 2021). These high pressure may lead to cavitation-the formation of bubbles-which may consequently block the propagation of ultrasound into the target (Lea-Banks and Hynynen, 2021).

This study has at least four limitations. First, we tested the effects of ultrasound frequency and pressure, but not the ultrasound waveform. For instance, we have not tested the effects of the duty cycle, instead using a fixed value of $10 \%$. Increases in the duty cycle are known to lead to stronger, cumulative effects (Airan et al., 2017; Zhong et al., 2019). Second, we quantified the release of a single drug, propofol. We used this drug for two reasons: 1) it is commonly applied in the clinics for neuromodulatory applications (Orser et al., 1994), and 2) there is an established optical procedure for the quantification of its release (Zhong et al., 2019). This limitation is mitigated by previous studies that demonstrated that polymer-based nanoparticles could encapsulate a variety of hydrophobic drugs (Liu et al., 2020). The more hydrophobic a drug, the higher its efficacy of 
encapsulation (Zhong et al., 2019). Using tri-block copolymers (hydrophilic-hydrophobic-hydrophilic), it is also possible to encapsulate hydrophilic drugs (Tian et al., 2012; Brzeziński and Biela, 2015; Grossen et al., 2017). Third, we quantified the release of nanoparticles using a single copolymer shell (PEG:PDLLA). This limitation is mitigated by a previous study, which found little variability in the release due to specific forms of the polymeric shell (Zhong et al., 2019). And fourth, the safety, blood clearance, and organ distribution investigations were only performed as a one-time procedure. More data, including repeated administrations, should be collected - especially in primates. Addressing these limitations lays out our next steps.

\section{Conclusions}

In summary, we found that nanoparticles filled with high-boiling point PFCs can be effectively activated with ultrasound at low $(300 \mathrm{kHz})$ frequencies. The effect is consistent with a mechanical expansion of the core. In addition, we found that such nanoparticles can be safely injected into the bloodstream of primates, have a half-life on the order of dozens of minutes, and are degraded mainly in the liver. This study informs the use of specific PFC-based nanoparticles and ultrasound parameters for effective, safe, and targeted drug release in humans.

\section{Materials and Methods}

\section{Materials}

Methoxy poly(ethylene glycol)-b-poly(D,L-lactide) (PEG-PDLLA) co-polymers with 2,000: 2,200 g/mol molecular weights, respectively, were obtained from PolyScitech (USA). $2 \mathrm{H}, 3 \mathrm{H}$ - decafluoropentane and perfluorooctyl bromide were obtained from Tokyo Chemical Industry Co. (Japan). Perfluoro-n-pentane was obtained from Strem Chemicals (USA). Propofol was obtained from Sigma Aldrich (Millipore Sigma, Canada). Infrared dye IR800RS NHS Ester was obtained from LI-COR Biosciences (USA). HPLC-grade tetrahydrofuran (THF) and methanol were obtained from Fisher Scientific (USA). Phosphate buffer solution (PBS) was obtained from Gibco (Thermo Fisher Scientific, USA).

\section{Nanoparticle production}

The process of manufacturing the drug-encapsulating, ultrasound-responsive PFC particles is illustrated at a conceptual level in (Figure 8) and described in detail in previous studies (Rapoport et al., 2007; Zhong et al., 2019). The process converts small $(<30 \mu \mathrm{m})$ micelles into much larger $(>300 \mu \mathrm{m})$ PFC-filled nanoparticles. First, the PEG-PDLLA polymer constituting the basis of the nanoparticle shell is dissolved in THF at a rate of $1 \mathrm{~mL}$ THF : $16 \mathrm{mg}$ polymer. For the biodistribution and blood clearance studies, infrared dye is added at a ratio of 1:32 (dye:polymer) for the rats and marmoset and 1:110 for the macaque. THF is then evaporated under vacuum until a gel-like layer remains. PBS is added at a rate of $1 \mathrm{~mL}$ PBS : $8 \mathrm{mg}$ polymer and placed on a shaker table at $120 \mathrm{rpm}$ to dissolve for 15 minutes. 
The addition of PBS orients the hydrophilic copolymer, PEG, toward the water and the hydrophobic, PDLLA, copolymer away from the water, and as a consequence, micelles are formed. Next, the PFC core and propofol are added and emulsified. A ratio of $1 \mathrm{mg}$ propofol : $2 \mathrm{mg}$ polymer was used in all cases. The nanoparticles' diameter can be controlled by the ratio of PFC to polymer, as reported previously (Rapoport et al., 2011). For PFOB and DFP nanoparticles, a ratio of $4.5 \mu \mathrm{L}$ PFC : $1 \mathrm{mg}$ polymer was used. The ratio for PFP was scaled up to $6.25 \mu \mathrm{L}: 1 \mathrm{mg}$ to account for PFC lost to vaporization before being emulsified. A $20 \mathrm{kHz}, 500 \mathrm{~W}$ sonicator with a cup horn attachment (VCX500, Sonics) was used to perturb the thermodynamic equilibrium of the micellar system, which leads to the incorporation of PFOB into the micelles and the formation of stable nanodroplets or nanoparticles (Gupta et al., 2015). The PFC and propofol are added to $15 \mathrm{~mL}$ centrifuge tubes and gently shaken to combine before adding $8 \mathrm{~mL}$ of the micelle solution. The samples are then sonicated in a cold bath at $20 \%$ power in 30-second intervals until the solution is cloudy and drug and PFC are fully emulsified (between 60 and 90 seconds in total). A custom temperature-controlled cooling system maintained the bath temperature during sonication at $2^{\circ} \mathrm{C}$ for PFP and $10^{\circ} \mathrm{C}$ for DFP and PFOB. PFP must be kept colder to minimize vaporization before emulsification, while DFP and PFOB require higher temperatures to emulsify successfully. We found this controlled temperature approach to maximize the consistency of the nanoparticle sizes, drug encapsulation, and release properties. The resulting solution contains the desired nanoparticles in addition to remaining micelles, dissolved polymer, and free propofol. Nanoparticles are isolated using three cycles of centrifugation at 3,000 relative centrifugal force (RCF) at $4^{\circ} \mathrm{C}$. After each cycle, the supernatant is discarded and the pellet dissolved in $5 \mathrm{~mL}$ fresh PBS. If the resulting solution contains larger particles than needed, these were removed by a slower centrifuge cycle for 1 minute at $800 \mathrm{RCF}$, this time keeping the supernatant. Larger particles contained in the pellet are discarded. For animal experiments, the nanoparticle solutions were sterilized for 3 hours under UV light, a protocol previously shown to yield effective sterilization (Tapia-Guerrero et al., 2020). Sterilization was conducted in glass vials in a custom chamber with an 8W UV lamp (Philips, USA).

\section{Nanoparticle characterization}

The sizes were measured using a Zetasizer Nano S (Malvern Panalytical, UK), which reports the intensityweighted size distribution. The size values reported in the Results section describe the mean \pm standard deviation of the distribution of the intensity values measured by the device. To quantify the amount of drug encapsulated, a $50 \mu \mathrm{L}$ solution of nanoparticles is added to $450 \mu \mathrm{L}$ of methanol to dissolve all components (Airan et al., 2017). A UV-Vis spectrophotometer (NanoDrop 2000, Thermo Scientific) is used to quantify the concentration by comparing the absorbance at $276 \mathrm{~nm}$ to a propofol standard curve.

\section{Apparatus}

As in a previous study (Zhong et al., 2019), drug release is quantified in standard $1.5 \mathrm{~mL}$ microcentrifuge tubes. Each tube with freshly produced nanoparticles is placed into a plastic holder. A focused ultrasonic transducer (H-115, $64 \mathrm{~mm}$ diameter, $52 \mathrm{~mm}$ focal depth, Sonic Concepts) was positioned $52 \mathrm{~mm}$ below the holder so the sample was within the ultrasound focus. Degassed water (AIMS III system with 


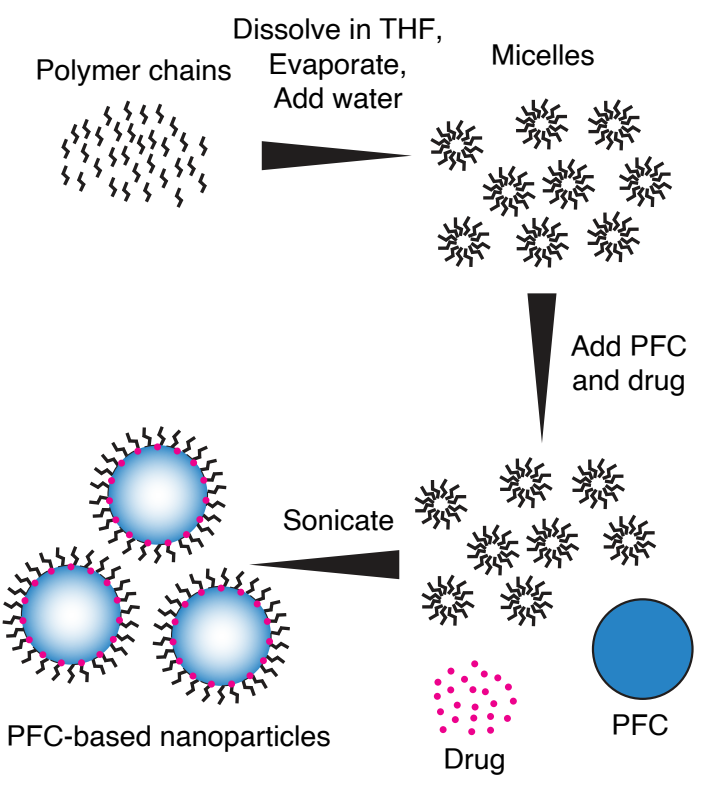

Figure 8. Production of the nanoparticles. The conversion of polymeric micelles into PFC-core-based nanoparticles using ultrasound. The three steps are described in detail in the text.

AQUAS-10 Water Conditioner, Onda) mediated coupling between the ultrasound face and the vial. The transducer was operated at $300 \mathrm{kHz}$ and the third harmonic, $900 \mathrm{kHz}$. Stimuli were generated using a function generator (33520b, Keysight). The signals were amplified using a 55- $\mathrm{dB}, 300 \mathrm{kHz}-30 \mathrm{MHz}$ power amplifier (A150, Electronics \& Innovation).

\section{Ultrasound parameters}

The ultrasound carrier frequencies for in vitro experiments were $300 \mathrm{kHz}$ and $900 \mathrm{kHz}$. Continuous pulses $100 \mathrm{~ms}$ in duration were repeated once per second for a total of 60 seconds (Airan et al., 2017; Zhong et al., 2019). The pressure levels at the vial location, measured in degassed water, were $0,0.5,0.9$, $1.3,1.7,2.1$, and $2.5 \mathrm{MPa}$. The pressure fields were measured using a capsule hydrophone (HGL-0200, Onda) calibrated between $250 \mathrm{kHz}$ and $40 \mathrm{MHz}$ and secured to 3-degree-of-freedom programmable translation system (Aims III, Onda).

For in vivo experiments, a similar ultrasound protocol was used to control for changes undergone by the particles as a result of sonication. The same transducer was operated at $248 \mathrm{kHz}$ in 60 -second blocks of $100 \mathrm{~ms}$ pulses. The animals were shaved and the transducer was coupled to the scalp using a $2 \%$ agar cone and ultrasound gel. The maximum pressure was estimated to be $1 \mathrm{MPa}$ for all animals. The pressure was estimated from free-field measurements using results from past studies, which indicate a transmission rate of 76\% through the skull of a 780g rat (O'Reilly et al., 2011) and 66\% through a macaque skull (Constans et al., 2017). Transmission through the marmoset skull has been less thoroughly studied but is likely similar to rats since they are close to the same weight. For further efficacy studies, pressure at the focus should be more carefully estimated. Ultrasound was applied to the rats at the midline, $2 \mathrm{~mm}$ posterior to the eyes for 60 seconds 5 minutes after administration of nanoparticles. For the marmoset, ultrasound was applied at the posterior surface of the skull for 60 seconds 5 minutes after each of the two administrations. The right and left visual cortex were targeted independently for the 
macaque. Ultrasound was applied in 60-second blocks over 90 minutes for a total of 4 sonication blocks per side starting 2 minutes after nanoparticle administration. Sonication of the left and right sides was interleaved.

\section{Drug release characterization}

$100 \mu \mathrm{L}$ of hexane was placed on top of $200 \mu \mathrm{L}$ nanoparticle solutions prior to sonication to act as a sink for released propofol (Airan et al., 2017). After 1 minute, 45 seconds of total incubation time, 50uL of hexane was extracted. The amount of dissolved propofol was quantified using UV-Vis spectrophotometry as described previously. The percent release efficacy is defined as the amount of the drug released into the hexane relative to the amount encapsulated. Each datapoint in Figure 4 included 3-4 distinct samples.

\section{Subjects}

An adult male old world monkey (Macaca mulatta), an adult male new world monkey (Callithrix jacchus), and 3 adult male Sprague-Dawley rats participated in the safety and biodegradability experiments. All procedures were approved by the Institutional Animal Care and Use Committee of the University of Utah.

\section{Animal procedures}

For the vital sign experiments, the rats were anesthetized with $80 \mathrm{mg} / \mathrm{kg}$ ketamine and $10 \mathrm{mg} / \mathrm{kg}$ xylazine administered intraperitoneally. A dose of sterilized PFOB nanoparticles containing $0.5 \mathrm{mg} / \mathrm{kg}$ propofol was administered to the first animal and $1 \mathrm{mg} / \mathrm{kg}$ to the subsequent animals. A total of $0.48,0.53$, and $1.1 \mathrm{ml}$ of the nanoparticle mixture was injected into the tail vein of the 3 animals. Biodistribution experiments were carried out one week later. The animals were anesthetized with $2.5-3 \%$ isoflurane, and dye-loaded nanoparticles were administered at a dose of $1 \mathrm{mg} / \mathrm{kg}$ propofol followed by an equal volume of sterile saline. After one hour, the animals were euthanized by coronary exsanguination under $5 \%$ isoflurane anesthesia.

The primates were preanesthetized with ketamine $(25 \mathrm{mg} / \mathrm{kg}$ intramuscularly) and intubated with endotracheal tubes. They were artificially ventilated and anesthesia maintained with $1-4 \%$ isoflurane throughout the procedure by veterinary staff. The animals were placed on warmed operating table pads to maintain body temperature. For the marmoset, dye-loaded nanoparticles were injected through the tail vein at doses of $1 \mathrm{mg} / \mathrm{kg}$ propofol for each of two injections separated by 45 minutes. A total volume of $2 \mathrm{ml}$ of nanoparticle solution was administered, followed by an equal volume of sterile saline. The marmoset was euthanized by an overdose of sodium pentobarbital and perfused transcardially with $4 \%$ paraformaldehyde 82 minutes after the first injection. For the macaque, one injection of dye-loaded nanoparticles was administered in the right saphenous vein at $1 \mathrm{mg} / \mathrm{kg}$ and a volume of $3 \mathrm{~mL}$, followed by an equal volume of sterile saline. Blood samples were taken from the left saphenous vein 2, 10, $20,40,80$, and 120 minutes following tail vein injection, at a volume of $1 \mathrm{~mL}$ each. Following 120 
minutes of monitoring, the macaque was euthanized by an overdose of sodium pentobarbital and perfused transcardially with $4 \%$ paraformaldehyde.

The procedures were painless and wholly consistent with the recommendations of the Panel on Euthanasia of the American Veterinary Medical Association. In all cases, heart rate was recorded using a portable ECG-monitoring system with electrode pads placed on the upper right and upper left upper abdomen and a reference on the lower left abdomen.

\section{Measurements of blood clearance and biodistribution}

Following the euthanasia, major organs were extracted and subjected to infrared fluorescence analysis using an IVIS system (Perkin Elmer) with methods similar to those previously demonstrated (Airan et al., 2017). Excitation was set to $745 \mathrm{~nm}$ and emission to $820 \mathrm{~nm}$, with 5 seconds of exposure. Extracted organs were placed directly on a dish in the field of view of the camera. In the macaque, the same organs as in the smaller animals were available, with the exception of the brain. Blood samples were pipetted into drops of $100 \mathrm{uL}$ on a dish. Total fluorescence for each of these samples was quantified using Aura software (Spectral Instruments), defining regions of interest incorporating the whole organ or blood sample and subtracting a background region of the same size. Percent biodistribution was computed as the total fluorescence of the region containing the organ divided by the sum of the fluorescence of all organs. Nanoparticle concentration in the blood was computed as the amount of fluorescence from each sample relative to the first sample, obtained at 2 minutes.

\section{Acknowledgments}

We thank Prof. Natalya Rapoport for her valuable input throughout the study and Sarah Haslam for assistance. This work was supported by the National Institute of Neurological Disorders and Stroke, grant R00NS100986, and by the National Institute of Mental Health, grant F32MH123019. 
Airan RD, Meyer RA, Ellens NP, Rhodes KR, Farahani K, Pomper MG, Kadam SD, Green JJ (2017) Noninvasive targeted transcranial neuromodulation via focused ultrasound gated drug release from nanoemulsions. Nano Letters 17:652-659.

Al-Harbi KS (2012) Treatment-resistant depression: therapeutic trends, challenges, and future directions. Patient preference and adherence 6:369.

Apfel RE, Holland CK (1991) Gauging the likelihood of cavitation from short-pulse, low-duty cycle diagnostic ultrasound. Ultrasound in medicine \& biology 17:179-185.

Bobo D, Robinson KJ, Islam J, Thurecht KJ, Corrie SR (2016) Nanoparticle-based medicines: a review of fda-approved materials and clinical trials to date. Pharmaceutical research 33:2373-2387.

Boissenot T, Bordat A, Larrat B, Varna M, Chacun H, Paci A, Poinsignon V, Fattal E, Tsapis N

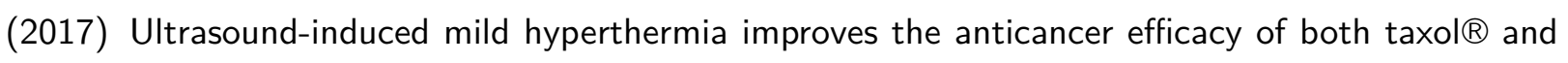
paclitaxel-loaded nanocapsules. Journal of Controlled Release 264:219-227.

Brzeziński M, Biela T (2015) Micro-and nanostructures of polylactide stereocomplexes and their biomedical applications. Polymer International 64:1667-1675.

Bystritsky A (2006) Treatment-resistant anxiety disorders. Molecular psychiatry 11:805-814.

Cao Y, Chen Y, Yu T, Guo Y, Liu F, Yao Y, Li P, Wang D, Wang Z, Chen Y et al. (2018) Drug release from phase-changeable nanodroplets triggered by low-intensity focused ultrasound. Theranostics 8:1327.

Castro Cl, Briceno JC (2010) Perfluorocarbon-based oxygen carriers: review of products and trials. Artificial organs 34:622-634.

Cobbold RS (2006) Foundations of biomedical ultrasound Oxford university press.

Cohn CS, Cushing MM (2009) Oxygen therapeutics: perfluorocarbons and blood substitute safety. Critical care clinics 25:399-414.

Constans C, Deffieux T, Pouget P, Tanter M, Aubry JF (2017) A 200-1380-khz quadrifrequency focused ultrasound transducer for neurostimulation in rodents and primates: Transcranial in vitro calibration and numerical study of the influence of skull cavity. IEEE transactions on ultrasonics, ferroelectrics, and frequency control 64:717-724.

Doinikov AA, Sheeran PS, Bouakaz A, Dayton PA (2014) Vaporization dynamics of volatile perfluorocarbon droplets: a theoretical model and in vitro validation. Medical physics 41:102901.

Elias WJ, Huss D, Voss T, Loomba J, Khaled M, Zadicario E, Frysinger RC, Sperling SA, Wylie S, Monteith SJ et al. (2013) A pilot study of focused ultrasound thalamotomy for essential tremor. New England Journal of Medicine 369:640-648. 
Elias WJ, Lipsman N, Ondo WG, Ghanouni P, Kim YG, Lee W, Schwartz M, Hynynen K, Lozano AM, Shah BB et al. (2016) A randomized trial of focused ultrasound thalamotomy for essential tremor. New England Journal of Medicine 375:730-739.

Emanuel NNM, Buchachenko AL (1987) Chemical physics of polymer degradation and stabilization, Vol. 1 VSP.

FDA (2019) Marketing clearance of diagnostic ultrasound systems and transducers. Food and Drug Administration FDA-2017-D-5372.

Grossen P, Witzigmann D, Sieber S, Huwyler J (2017) Peg-pcl-based nanomedicines: A biodegradable drug delivery system and its application. Journal of Controlled Release 260:46-60.

Gupta R, Shea J, Scaife C, Shurlygina A, Rapoport N (2015) Polymeric micelles and nanoemulsions as drug carriers: therapeutic efficacy, toxicity, and drug resistance. Journal of Controlled Release 212:70-77.

Jaffe DH, Rive B, Denee TR (2019) The humanistic and economic burden of treatment-resistant depression in europe: a cross-sectional study. BMC psychiatry 19:247.

Krafft MP (2001) Fluorocarbons and fluorinated amphiphiles in drug delivery and biomedical research. Advanced drug delivery reviews 47:209-228.

Kripfgans OD, Fowlkes JB, Miller DL, Eldevik OP, Carson PL (2000) Acoustic droplet vaporization for therapeutic and diagnostic applications. Ultrasound in medicine \& biology 26:1177-1189.

Lea-Banks H, O'Reilly M, Hynynen K (2019) Ultrasound-responsive droplets for therapy: A review. Journal of Controlled Release 293:144-154.

Lea-Banks H, Hynynen K (2021) Sub-millimetre precision of drug delivery in the brain from ultrasoundtriggered nanodroplets. Journal of Controlled Release 338:731-741.

Lea-Banks H, Meng Y, Wu SK, Belhadjhamida R, Hamani C, Hynynen K (2021) Ultrasound-sensitive nanodroplets achieve targeted neuromodulation. Journal of Controlled Release 332:30-39.

Li X, Sui Z, Li X, Xu W, Guo Q, Sun J, Jing F (2018) Perfluorooctylbromide nanoparticles for ultrasound imaging and drug delivery. International journal of nanomedicine 13:3053.

Liu Y, Yang G, Baby T, Chen D, Weitz DA, Zhao CX (2020) Stable polymer nanoparticles with exceptionally high drug loading by sequential nanoprecipitation. Angewandte Chemie 132:4750-4758.

Louis ED, Rios E, Henchcliffe C (2010) How are we doing with the treatment of essential tremor (et)? persistence of patients with et on medication: data from 528 patients in three settings. European journal of neurology 17:882-884.

Lyons KE, Pahwa R (2004) Deep brain stimulation and essential tremor. Journal of clinical neurophysiology 21:2-5. 
Needham D, Dewhirst MW (2001) The development and testing of a new temperature-sensitive drug delivery system for the treatment of solid tumors. Advanced drug delivery reviews 53:285-305.

Orser B, Wang L, Pennefather P, MacDonald J (1994) Propofol modulates activation and desensitization of gabaa receptors in cultured murine hippocampal neurons. Journal of Neuroscience 14:7747-7760.

O'Reilly MA, Muller A, Hynynen K (2011) Ultrasound insertion loss of rat parietal bone appears to be proportional to animal mass at submegahertz frequencies. Ultrasound in medicine \& biology 37:1930-1937.

Rapoport N (2012) Phase-shift, stimuli-responsive perfluorocarbon nanodroplets for drug delivery to cancer. Wiley Interdisciplinary Reviews: Nanomedicine and Nanobiotechnology 4:492-510.

Rapoport N (2016) Drug-loaded perfluorocarbon nanodroplets for ultrasound-mediated drug delivery In Therapeutic Ultrasound, pp. 221-241. Springer.

Rapoport N, Christensen DA, Kennedy AM, Nam KH (2010) Cavitation properties of block copolymer stabilized phase-shift nanoemulsions used as drug carriers. Ultrasound in medicine \& biology 36:419-429.

Rapoport N, Gao Z, Kennedy A (2007) Multifunctional nanoparticles for combining ultrasonic tumor imaging and targeted chemotherapy. Journal of the National Cancer Institute 99:1095-1106.

Rapoport N, Gupta R, Kim YS, O'Neill BE (2015) Polymeric micelles and nanoemulsions as tumortargeted drug carriers: Insight through intravital imaging. Journal of Controlled Release 206:153-160.

Rapoport N, Nam KH, Gupta R, Gao Z, Mohan P, Payne A, Todd N, Liu X, Kim T, Shea J et al. (2011) Ultrasound-mediated tumor imaging and nanotherapy using drug loaded, block copolymer stabilized perfluorocarbon nanoemulsions. Journal of Controlled Release 153:4-15.

Rapoport NY, Kennedy AM, Shea JE, Scaife CL, Nam KH (2009) Controlled and targeted tumor chemotherapy by ultrasound-activated nanoemulsions/microbubbles. Journal of Controlled Release 138:268-276.

Riis T, Kubanek J (2021) Effective ultrasonic stimulation in human peripheral nervous system. IEEE Transactions on Biomedical Engineering .

Sheeran PS, Luois SH, Mullin LB, Matsunaga TO, Dayton PA (2012) Design of ultrasonically-activatable nanoparticles using low boiling point perfluorocarbons. Biomaterials 33:3262-3269.

Sheeran PS, Matsunaga TO, Dayton PA (2013) Phase-transition thresholds and vaporization phenomena for ultrasound phase-change nanoemulsions assessed via high-speed optical microscopy. Physics in Medicine \& Biology 58:4513.

Shpak O, Verweij M, Vos HJ, de Jong N, Lohse D, Versluis M (2014) Acoustic droplet vaporization is initiated by superharmonic focusing. Proceedings of the National Academy of Sciences 111:1697-1702. 
Somaglino L, Mousnier L, Giron A, Urbach W, Tsapis N, Taulier N (2021) In vitro evaluation of polymeric nanoparticles with a fluorine core for drug delivery triggered by focused ultrasound. Colloids and Surfaces B: Biointerfaces 200:111561.

Tapia-Guerrero Y, Prado-Audelo D, Borbolla-Jiménez F, Giraldo Gomez D, García-Aguirre I, Colín-Castro C, Morales-González J, Leyva-Gómez G, Magaña J et al. (2020) Effect of uv and gamma irradiation sterilization processes in the properties of different polymeric nanoparticles for biomedical applications. Materials 13:1090.

Tian H, Tang Z, Zhuang X, Chen X, Jing X (2012) Biodegradable synthetic polymers: Preparation, functionalization and biomedical application. Progress in Polymer Science 37:237-280.

Wang JB, Aryal M, Zhong Q, Vyas DB, Airan RD (2018) Noninvasive ultrasonic drug uncaging maps whole-brain functional networks. Neuron 100:728-738.

Wu SY, Fix SM, Arena CB, Chen CC, Zheng W, Olumolade OO, Papadopoulou V, Novell A, Dayton PA, Konofagou EE (2018) Focused ultrasound-facilitated brain drug delivery using optimized nanodroplets: vaporization efficiency dictates large molecular delivery. Physics in Medicine \& Biology 63:035002.

Zesiewicz TA, Chari A, Jahan I, Miller AM, Sullivan KL (2010) Overview of essential tremor. Neuropsychiatric disease and treatment 6:401.

Zhang M, Fabiilli ML, Haworth KJ, Fowlkes JB, Kripfgans OD, Roberts W, Ives K, Carson PL (2010) Initial investigation of acoustic droplet vaporization for occlusion in canine kidney. Ultrasound in medicine \& biology 36:1691-1703.

Zhong Q, Yoon BC, Aryal M, Wang JB, llovitsh T, Baikoghli M, Hosseini-Nassab N, Karthik A, Cheng $\mathrm{R}$, Ferrara $\mathrm{K}$ et al. (2019) Polymeric perfluorocarbon nanoemulsions are ultrasound-activated wireless drug infusion catheters. Biomaterials 206:73-86. 